\title{
THE RELATIONSHIP BETWEEN DRIVING BEHAVIOR AND ENTROPY
}

\author{
Jeffrey D. Dawson, Joshua D. Cosman, Yang Lei, \\ Elizabeth Dastrup, JonDavid Sparks \\ Dept. of Biostatistics \\ Matthew Rizzo \\ Department of Neurology \\ University of Iowa \\ E-mail: jeffrey-dawson@uiowa.edu
}

PAPER NOT AVAILABLE

\begin{abstract}
\section{OBJECTIVES}

High variability in the lateral control of a vehicle may result in an increased likelihood of accidents. Boer (2000) proposed a method of quantifying variability in steering wheel position, termed "entropy" (scaled between 0 and 1). In this approach, the steering wheel position at each time point is predicted based on the position at the three preceding time points, and the discrepancies between the predicted and observed values are utilized to define a baseline distribution of prediction errors within a subject. This distribution is then used as a reference for calculating a summary "entropy" metric in follow-up segments of driving, such as when a driver may be distracted when using a cell phone. This same concept has also been applied to the lateral position of a vehicle (Dawson et al., 2006). The objective of this study was to ascertain whether entropy was affected by behavioral factors such as steering techniques and speed. We hoped to gain insight regarding the usefulness of entropy measures, and the appropriate interpretation of statistical tests based on entropy.
\end{abstract}

\section{METHODS}

We designed a simple driving route in a simulator known as SIREN (Rizzo, 2004), with a straight road segment of $3.7 \mathrm{~km}$, followed by an S-curved road segment of $3.8 \mathrm{~km}$. Using an expert driver familiar with the simulator, we performed a factorial experiment with different steering techniques (normal driving, swerving, and steering using a rigid grip and sudden "jerks") and driving speeds (35 mph, $55 \mathrm{mph}$, and $75 \mathrm{mph}$ ). Data on steering wheel position and lane position were collected at 30 frames per second, and then reduced to 5-frame blocks of 167 msec each. Based on these blocks, we estimated the baseline parameter to characterize the prediction errors for each drive during the straight road section, and then applied this parameter to the straight and curved road sections in order to calculate entropy. This approach was used for both steering and lane position entropy. The data were analyzed using multiple linear regresssion to assess the affects of steering technique and speed, adjusting for road curvature. We also calculated Pearson correlation coefficients to measure the association between steering and lane position entropy. 


\section{RESULTS}

Data were obtained on a total of 40 drive segments. Steering entropy ranged from 0.34 to 0.90 , with a mean (SD) of $0.56(0.16)$. Lane position entropy ranged from 0.34 to 0.93 , with a mean (SD) of $0.61(0.15)$. Compared to normal driving, steering behavior involving jerking motions tended to lower the steering entropy by $0.14(\mathrm{p}=0.012)$, and tended to lower the lane position entropy by $0.25(\mathrm{p}<0.001)$. Swerving in wide lateral motions had no effect on steering entropy, and only a minor effect on lane position entropy, decreasing it by 0.07 . Compared to driving at $35 \mathrm{mph}$, driving at either $55 \mathrm{mph}$ or $75 \mathrm{mph}$ increased the steering entropy by an average of 0.08 , but had no effect on lane position entropy. Although not our primary focus, we found that driving in curved sections tended to have higher entropy measures (increase of 0.21 for steering and 0.20 for lane position; $p<0.001$ in both cases). Despite a few outliers, the correlation between steering and lane position entropy was found to be high $(\mathrm{r}=0.84$; see Figure 1$)$.

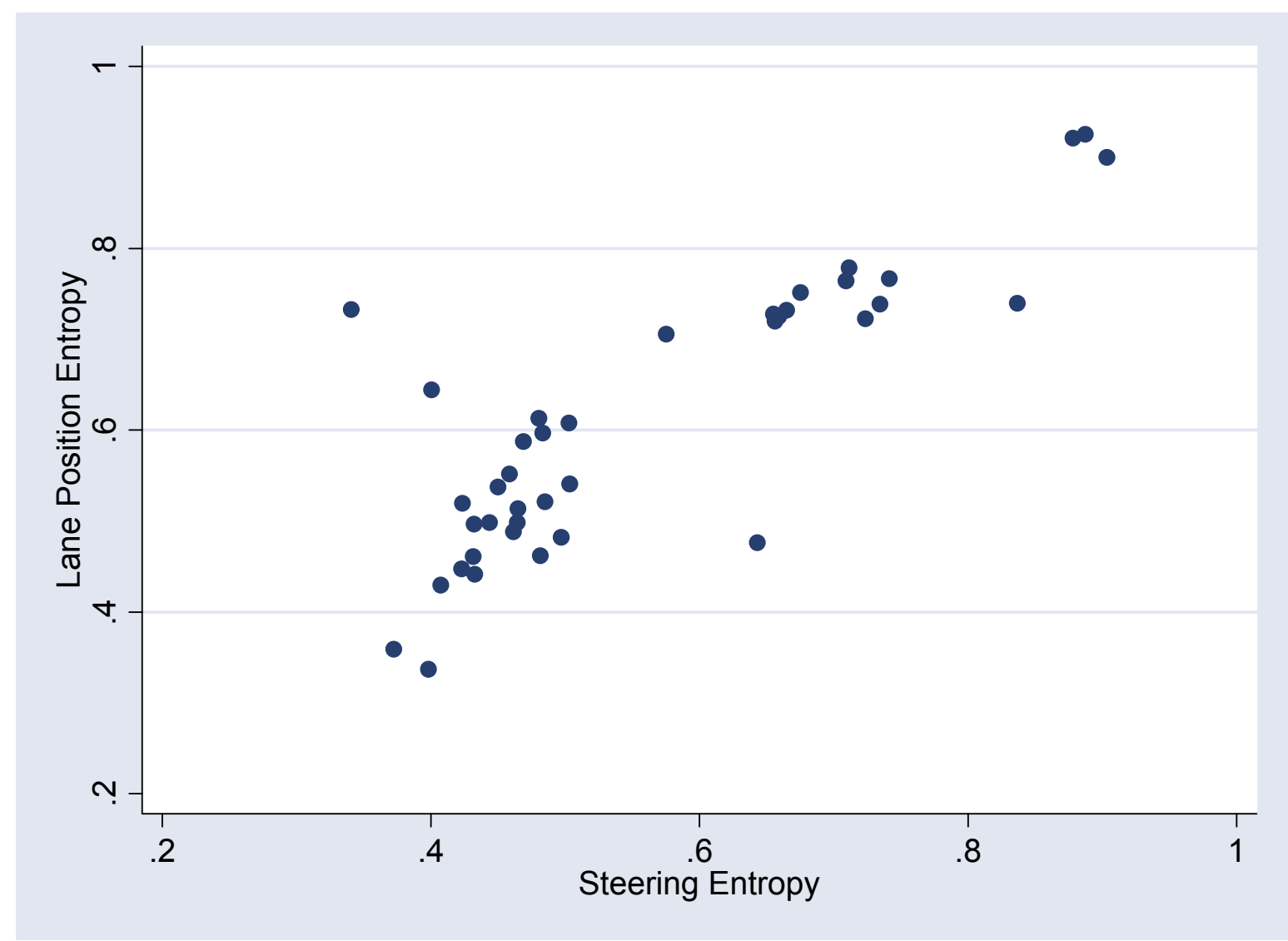

Figure 1. Relationship between Steering Entropy and Lane Position Entropy in 40 Segments

\section{CONCLUSIONS}

Although entropy is often considered as an increasing function of workload, and would presumably increase in non-optimal conditions, some unsafe driving behaviors are actually 
negatively associated with entropy. Safe driving often involves making frequent minor steering adjustments, especially in curved sections of the road, which might lead to an increase in the entropy measure. If a driver rigidly holds onto the steering wheel and then makes large corrections when approaching or crossing a lane boundary, the fixed steering wheel position over several seconds may actually cause an apparent decrease in entropy. In summary, entropy may be a useful tool in quantifying vehicular control, but caution should be exercised when interpreting the results, as the associations involving entropy are not always in the anticipated direction.

\section{REFERENCES}

Boer, E.R. (2000). Behavioral Entropy as an Index of Workload. 44th Annual Meeting of the Human Factors and Ergonomics Society (HFES2000), San Diego, CA, July 30 - August 4, 2000.

Dawson, J.D., Cavanaugh, J.E., Zamba, K.D., Rizzo, M. (2006). Measuring Lateral Control in Driving Studies. Biometric Society (ENAR) Annual Meeting, Tampa, FL, March 26-29, 2006.

Rizzo, M. (2004). Safe and unsafe driving. In: Rizzo M, Eslinger PJ, (Eds.). Principles and Practice of Behavioral Neurology and Neuropsychology. Philadelphia, Pennsylvania: WB Saunders, 197-222. 